\title{
Gamificação e aprendizagem de jovens: RBI sobre os Aspectos Cognitivos e o Design de Interfaces
}

Isabela Sousa Guimarães;

Luís Carlos Paschoarelli

resumo:

O presente artigo aborda a importância da ergonomia cognitiva na criação de ferramentas de gamificação e tem por propósito discutir a efetividade de sua aplicação no ensino (médio e superior) de jovens, bem como o seu impacto cognitivo. O método utilizado foi a Revisão Bibliográfica Integrativa (RBI), realizada entre os anos de 2010 a 2020, por meio da seleção de quatro bases eletrônicas, distribuídas entre os grupos de strings de busca: Gamification + Cognitive Impact + Young. Os quatro resultados obtidos da pesquisa - Silva, Sales e Castro (2019), Cabada (2018), Fan, Xiao e Su (2015) e Barata et. al. (2013) - possibilitaram compreender o uso da gamificação em plataformas de aprendizagem, seus estímulos para o aumento no número de acertos e os demais benefícios cognitivos trazidos pela inserção dessas ferramentas no contexto de ensino.

\section{palavras-chave:}

Gamificação; Design de interfaces; Ergonomia cognitiva; Impacto cognitivo; Revisão bibliográfica integrativa 


\section{Introdução}

A gamificação tem sido uma ferramenta poderosa no processo educacional de crianças, e é amplamente estudada por pesquisadores de todo mundo. A relação entre a gamificação e o processo de aprendizagem ativa é bem próxima. Além de fornecer melhorias relacionadas ao processo de ensino, existe uma série de outros estudos indicando melhorias de concentração (CSIKSZENTMIHALYI, 1990), empenho (MCCLEAN, 2001) condicionamento do estado emocional (LANDOWSKA, 2014) dentre muitas outras interferências positivas. De acordo com Norman (2008), "brincar serve para muitos propósitos. [...] Ajuda as crianças a desenvolverem uma mistura de cooperação e competição exigidas para viver de maneira eficiente em grupos sociais" (p. 155). Já para Johan Huizinga (2018), filósofo que estuda os processos de ludicidade, entende o ato de brincar como algo inerente, não apenas ao ser humano, como também a outros animais, e que é uma forma de treinamento para os desafios da vida real. $\mathrm{O}$ processo de aprender brincando atende às expectativas do referencial de aprendizagem construtivista (PIAGET, 1998), compreende o aspecto social, definitivo na visão de Vygotsky (2003), agrega valor na aprendizagem significativa de Ausubel (1963), dentre outras teorias de aprendizagem.

A experiência digital alinhada ao processo de ensino só cresce ao longo dos anos. Segundo Pesquisa realizada no ano de 2018 pelo Instituto Brasileiro de Geografia e Estatística-IBGE, a internet já era utilizada em $79,1 \%$ das casas brasileiras, e o uso do telefone celular, àquele tempo, já servia como o meio principal de acesso à Internet a praticamente todos os domicílios [99,2\%] (IBGE Educa, $\mathrm{s} / \mathrm{d})$. "No Brasil, existem cerca de 1,5 milhão de estudantes que optaram pela modalidade de ensino a distância - 18,6\% das matrículas totais. Em 2004, eram apenas 60 mil, 4,2\% do total" (SILVA, 2018) e esse movimento exigiu uma maior variedade de ferramentas voltadas ao ensino, como a sala de aula invertida, juri simulado, utilização de hipertexto e a aplicação de ferramentas de gamificação e demais jogos de interação virtual (SIMULARE, 2019) como complemento ou incentivador do processo de aprendizagem.

O ensino de jovens - indivíduos de 15 a 29 anos de acordo com o Art. 1o da Lei $\mathrm{n}^{\mathrm{o}} 12.852$, de 5 de agosto de 2013 do Estatuto da Juventude no Brasil (Brasil, 2013) — pode se beneficiar muito dessa interação entre gamificação e plataformas digitais interativas de aprendizagem, não apenas pelo seu potencial de melhoria do desempenho dos resultados obtidos em testes, como também dos vários outros benefícios cognitivos e interacionais que ela pode oferecer.

Por esse motivo, o presente estudo propôs aplicar uma Revisão Bibliográfica Integrativa para conhecer o estado da arte sobre as ferramentas de gamificação como utensílios na aprendizagem de jovens, usuários de plataformas digitais interativas; e sua contribuição para o desempenho cognitivo desses indivíduos.

\section{Procedimentos Metodológicos}

O presente estudo é de cunho teórico baseado em levantamento bibliográfico (BOTELHO, CUNHA, MACEDO, 2011) integralizado, cujo método sistemático utilizado foi o Prisma. Foram realizadas pesquisas em quatro bases de dados, através da identificação de estratégias de busca PICO - Figura 1 (METHLEY et. al., 2014).

Figura 1: Determinação do PICO segundo método sistemático Prisma.

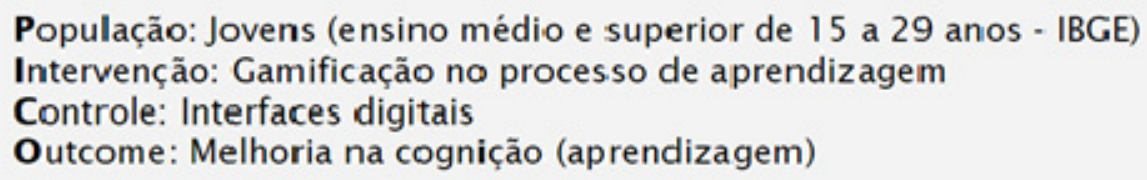

Fonte: Desenvolvido pelos autores.

Por meio dos descritores: (Gamification)* AND (Cognitive impact)* AND (Young)* foram selecionados artigos compatíveis com artigos científicos publicados em revistas científicas ou em 
congressos/conferências, nos idiomas inglês, português e espanhol e que fossem abertos ao público. A ferramenta utilizada para captação e o processo de seleção desses artigos foi o Mendeley.

Dentre os critérios para inclusão, encontravam-se avaliações que deveriam ser quantitativas no tocante à aprendizagem e procedimentos de gamificação que deveriam estar atrelados ao uso de alguma plataforma digital. Os estudos poderiam avaliar outras áreas relacionadas ao aspecto cognitivo (emoção, engajamento, entre outros), desde que possuíssem o fator aprendizado nos resultados quantitativos, fossem eles positivos ou negativos. Também poderiam avaliar professores e alunos, desde que as avaliações e testes fossem separados nos resultados quantitativos apresentados nos estudos e, por fim, era importante que os alunos avaliados estivessem matriculados em cursos de ensino médio ou superior.

\section{Resultados}

Iniciado o processo de avaliação dos trabalhos coletados nas bases de dados e dos registros adicionais, os 62 artigos coletados no total passaram por quatro crivos de seleção (Figura 2), sendo eles: retirada de artigos em duplicidade pela coleta das bases de dados, leitura do título, resumo e palavras-chave, leitura dos textos por completo, estudos incluídos em síntese qualitativa (meta-análise).

Figura 2: Processo de seleção de artigos.

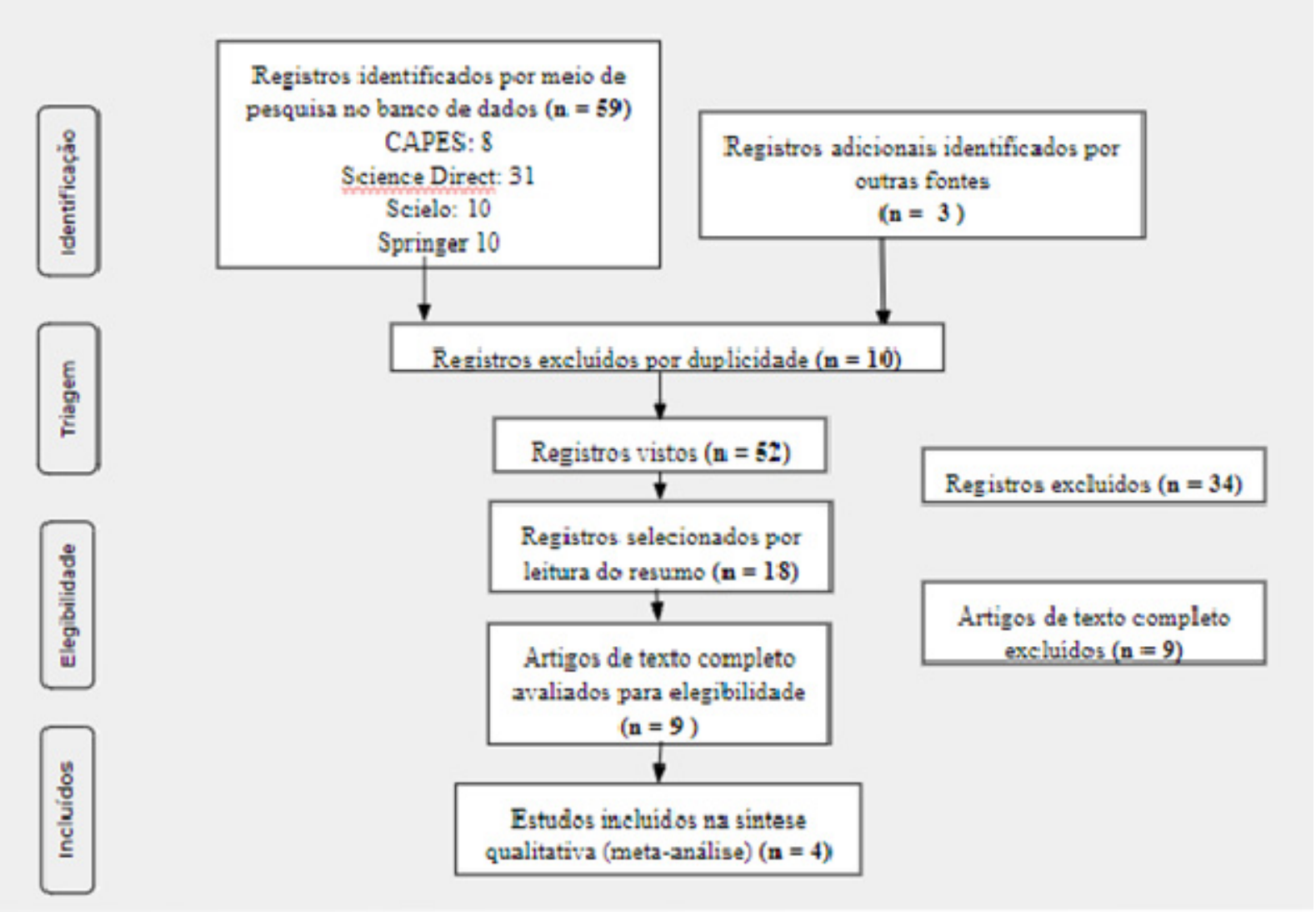

Fonte: Desenvolvido pelos autores.

Através da filtragem de resultados, quatro artigos foram selecionados a partir do foco de interesse do trabalho (Quadro 1).

Quadro 1 - Artigos selecionados para a discussão

\begin{tabular}{|c|c|c|l|l|l|l|}
\hline № & Autores & Ano & \multicolumn{1}{c|}{ Título } & Participantes & \multicolumn{1}{c|}{ População } & \multicolumn{1}{c|}{$\begin{array}{l}\text { Periódico ou } \\
\text { conferência }\end{array}$} \\
\hline 1 & $\begin{array}{l}\text { SILVA, } \\
\text { SALES, } \\
\text { CASTRO }\end{array}$ & 2019 & $\begin{array}{l}\text { Gamificação como } \\
\text { estratégia de } \\
\text { aprendizagem ativa } \\
\text { no ensino de Física }\end{array}$ & 49 & $\begin{array}{l}2^{\circ} \text { ano do E.M. } \\
\text { dos cursos de } \\
\text { Técnico } \\
\text { Integrado IFCE }\end{array}$ & $\begin{array}{l}\text { Revista } \\
\text { Brasileira de } \\
\text { Ensino de Física }\end{array}$ \\
\hline
\end{tabular}


Quadro 1 - Artigos selecionados para a discussão (continuação)

\begin{tabular}{|c|c|c|c|c|c|c|}
\hline 2 & CABADA & 2018 & $\begin{array}{l}\text { Reconocimiento } \\
\text { afectivo y } \\
\text { gamificación } \\
\text { aplicados al } \\
\text { aprendizaje de } \\
\text { Lógica algorítmica y } \\
\text { programación }\end{array}$ & 42 & $\begin{array}{l}\text { Estudantes de } \\
\text { Engenharia } \\
\text { Industrial }\end{array}$ & $\begin{array}{l}\text { REDIE - Revista } \\
\text { Electrónica de } \\
\text { Investigación } \\
\text { Educativa }\end{array}$ \\
\hline 3 & $\begin{array}{l}\text { FAN, } \\
\text { XIAO, SU }\end{array}$ & 2015 & $\begin{array}{l}\text { The Effects of } \\
\text { Learning Styles and } \\
\text { Meaningful Learning } \\
\text { on the Learning } \\
\text { Achievement of } \\
\text { Gamification Health } \\
\text { Education } \\
\text { Curriculum }\end{array}$ & 46 & $\begin{array}{l}1^{\circ} \text { Ano do } \\
\text { ensino médio }\end{array}$ & $\begin{array}{l}\text { Eurasia Journal } \\
\text { of Mathematics, } \\
\text { Science \& } \\
\text { Technology } \\
\text { Education }\end{array}$ \\
\hline 4 & $\begin{array}{l}\text { BARATA } \\
\text { et.al. }\end{array}$ & 2013 & $\begin{array}{l}\text { Improving } \\
\text { Participation and } \\
\text { Learning with } \\
\text { Gamification }\end{array}$ & $\begin{array}{l}52,62,41,35 \text { e } 52 \\
\text { (por ano) }\end{array}$ & $\begin{array}{l}\text { Estudantes de } \\
\text { MCP (cursos de } \\
\text { Sistemas de } \\
\text { informação e } \\
\text { Engenharia da } \\
\text { Computação) do } \\
\text { Instituto } \\
\text { Superior } \\
\text { Técnico. }\end{array}$ & $\begin{array}{l}\text { Gamification } \\
2013 \\
\text { (Conferência) }\end{array}$ \\
\hline
\end{tabular}

Fonte: Desenvolvido pelos autores.

O primeiro estudo (SILVA, SALES, CASTRO, 2019) investiga o potencial da aplicação de softwares e dinâmicas gamificadas para a melhoria na compreensão dos alunos na compreensão de fenômenos da Física, em específico a ótica geométrica, a reflexão da luz, fenômenos ópticos e formação de imagens em espelhos planos. O processo se resumia a sete etapas, sempre em comparativos em relação a atividades feitas pelo grupo controle: aula expositiva do conteúdo utilizando "ilhas de aprendizagem", verificação do conhecimento intrínseco do aluno por meio da plataforma gamificada Kahoot, exibição de documentários para fins de contextualização do tema de estudo proposto, a utilização da plataforma PHET para atividades relacionadas ao tema estudado, aulas experimentais em laboratório, utilização da plataforma Quizizz e revisão do conteúdo e dos questionários em sala de aula.

Figura 3: Plataforma digital gamificada de testes Kahoot.

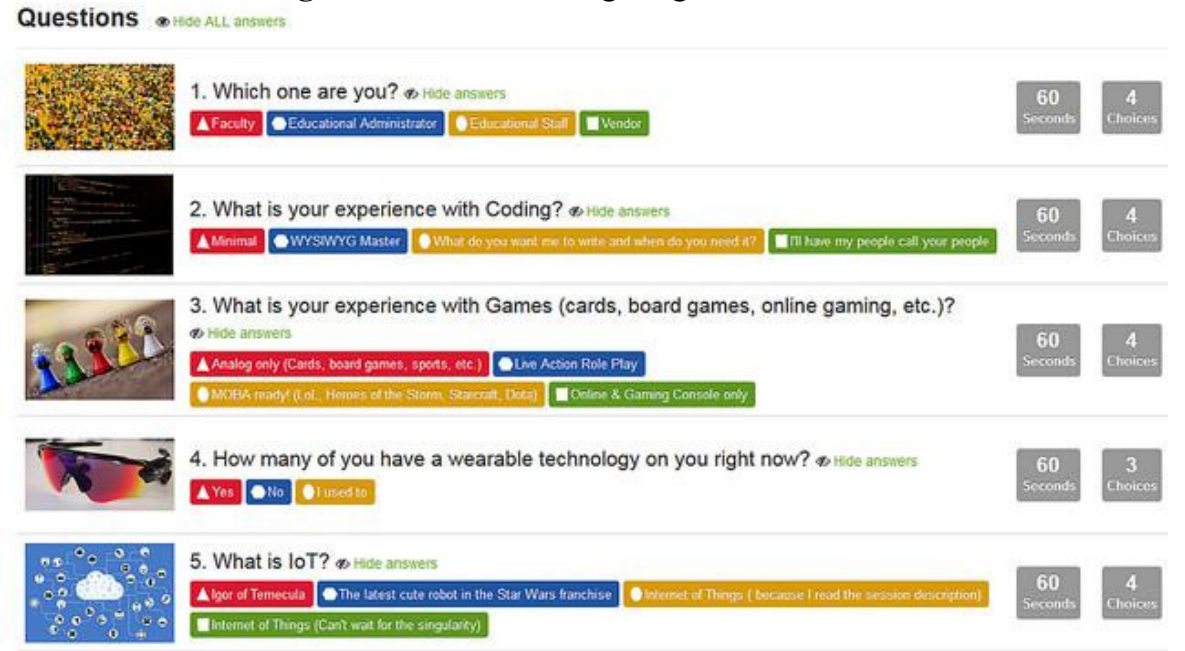

Fonte: ARREGUY, 2017. Acesso em 14 set 2020. 
O segundo estudo (CABADA, 2018) utiliza de uma plataforma digital interativa e gamificada para a observação de melhorias de aprendizado e de estado emocional durante o ensino de algoritmos e de lógica de programação. Para tanto, a plataforma utilizada foi a EasyLogic em cinco etapas: introdução ao motivo da avaliação, pré-teste, introdução à plataforma EasyLogic, utilização do software pelos alunos e exame pós-teste para avaliação dos resultados.

Figura 4: Exemplificação da utilização da EasyLogic por um aluno.

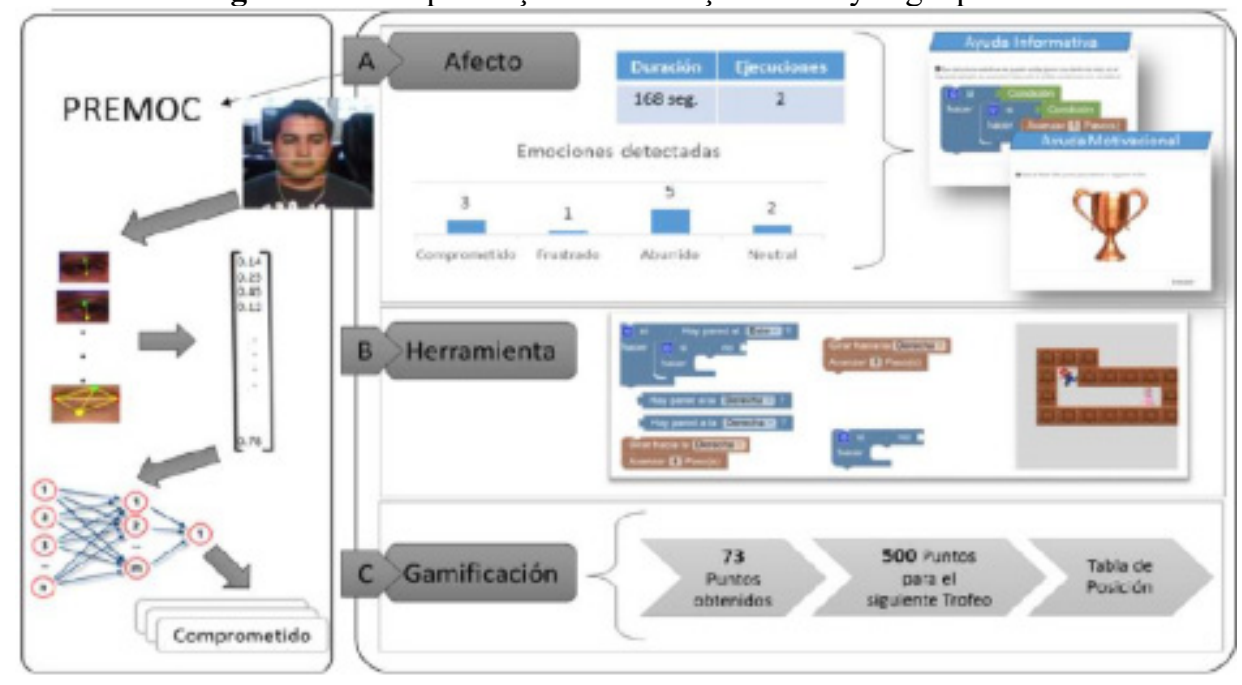

Fonte: CABADA, 2018.

O terceiro estudo (FAN, XIAO, SU, 2015) teve como objetivo principal o aprendizado gamificado e ativo sobre o sistema circulatório humano por estudantes do ensino médio. Para tal, foi desenvolvido um aplicativo especialmente para a aplicação dessas atividades de ensino, chamado Mobile Meaningful Blood Circulation Learning System, ou MMBCLS.

Figura 5: Atividades presentes no MMBCLS.

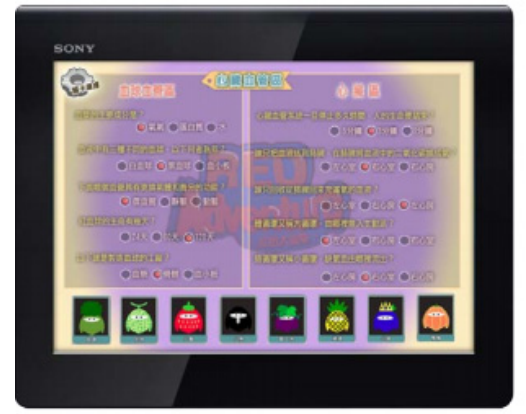

(a) Assessment 1. Cardiovascular circulation system

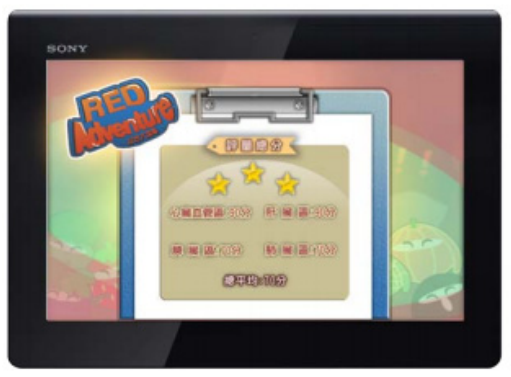

(c) Assessment 3. Lung circulation system

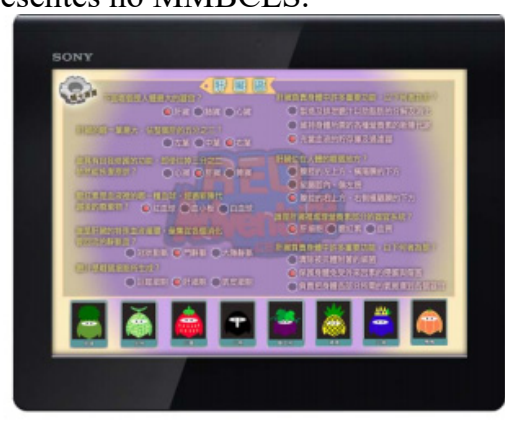

(b) Assessment 2. Liver circulation system

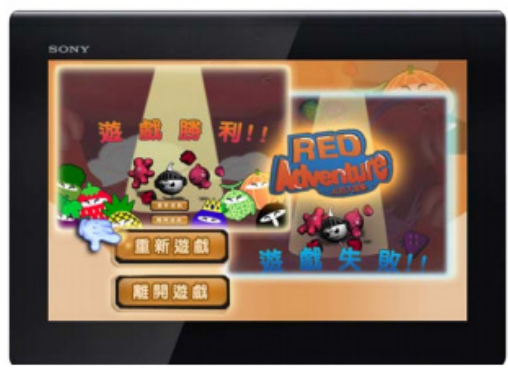

(d) Assessment 4. Spleen circulation system

Fonte: (FAN, XIAO, SU, 2015). 
Por último, o estudo quatro (BARATA et. al., 2013) trouxe valores a níveis institucionais, atribuindo ferramentas de gamificação ao Moodle aos alunos do curso de Sistemas de Informação e Engenharia da Computação da faculdade durante cinco turmas diferentes.

Figura 5: Leaderboard (ou pódio) disponível no Moodle para os alunos da instituição de ensino.

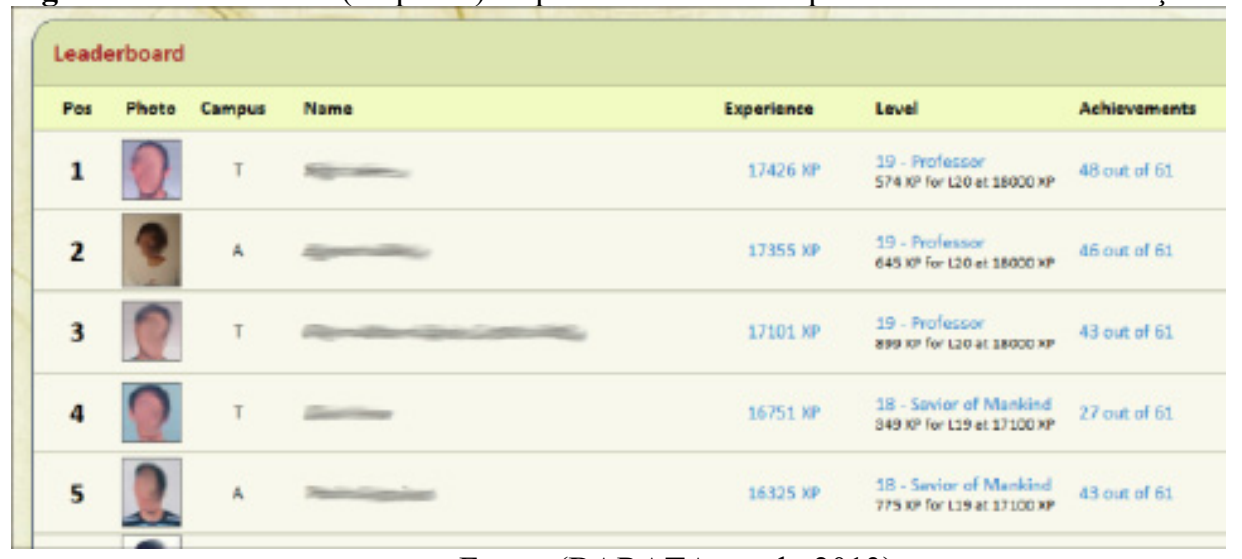

Fonte: (BARATA et. al., 2013).

Destaca-se que tanto os países dos trabalhos apresentados quanto as plataformas utilizadas como objetos de estudo foram diferentes, e que alguns estudos fizeram uso de mais de uma plataforma (Gráfico 1).

Gráfico 1: Informações referentes aos artigos selecionados.

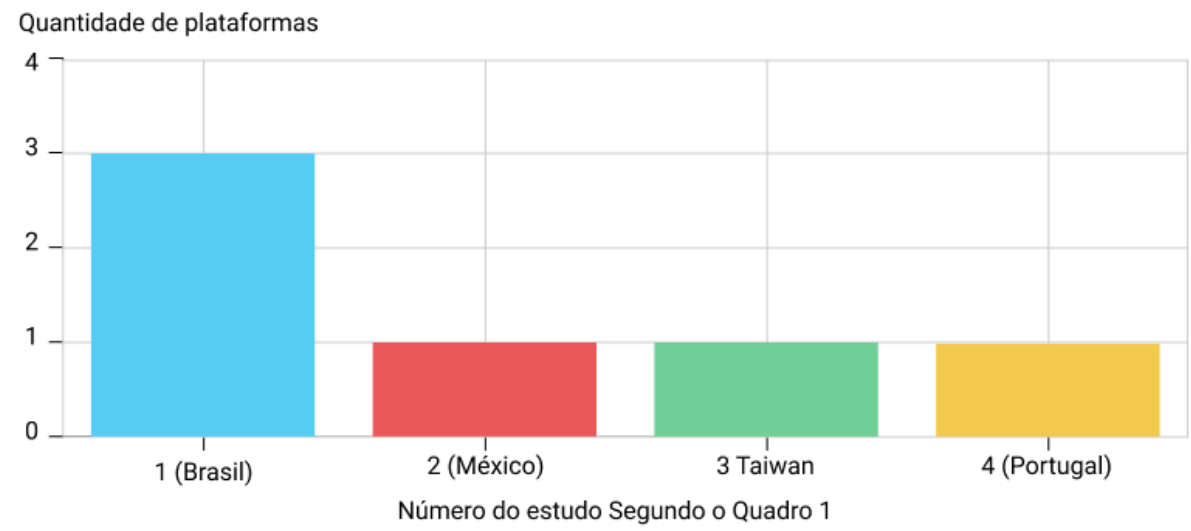

Fonte: Desenvolvido pelos autores.

Após a seleção de artigos, procurou-se analisar os resultados desses estudos que traziam atividades online de gamificação para alunos do ensino fundamental e médio. Percebe-se (Quadro 02) uma melhoria significativa no número de acertos das perguntas feitas em testes após a interação desses usuários com ferramentas de gamificação nas plataformas digitais aplicadas aos alunos, na maioria dos casos. $\mathrm{O}$ único estudo cujo resultado não evidenciou resultados significativos foi o número 2.

Quadro 2: Resultados no tocante ao impacto cognitivo no aprendizado de jovens pelo uso de gamificação em interfaces digitais de aprendizagem

\begin{tabular}{|l|l|l|}
\hline $\begin{array}{c}\text { № do } \\
\text { artigo }\end{array}$ & Autores & \multicolumn{1}{c|}{$\begin{array}{c}\text { Resultados no tocante ao impacto cognitivo no aprendizado de jovens em } \\
\text { plataformas digitais utilizando gamificação (melhoria do desempenho em } \\
\text { quantidades de acertos em testes) }\end{array}$} \\
\hline 1 & $\begin{array}{l}\text { SILVA, SALES, } \\
\text { CASTRO }\end{array}$ & $\begin{array}{l}\text { "A pesquisa evidenciou que a gamificação apresentou bom potencial para } \\
\text { promover a aprendizagem ativa pelos alunos" (SILVA, SALES, CASTRO, } \\
\text { 2019 p.8). } \\
\text { Aumento no número de acertos em } 20 \% \text { em comparação com o pré-teste e de } \\
13,21 \% \text { a mais que o grupo controle. } \\
\text { (Resumo dos resultados do ganho normatizado de Hake) }\end{array}$ \\
& &
\end{tabular}


Quadro 2: Resultados no tocante ao impacto cognitivo no aprendizado de jovens pelo uso de gamificação em interfaces digitais de aprendizagem

(Continuação)

\begin{tabular}{|l|l|l|}
\hline 2 & CABADA & $\begin{array}{l}\text { O impacto cognitivo esperado não foi alcançado pelo experimento. Os } \\
\text { resultados foram inferiores a 0,05\%. }\end{array}$ \\
\hline 3 & FAN, XIAO, SU & $\begin{array}{l}\text { "Tendo aplicado a estratégia de aprendizado móvel, significativa, o pós-teste } \\
\text { médio do grupo experimental aparece como 88, obviamente aumentado em 10, } \\
\text { enquanto o grupo controle não revela diferenças óbvias no pós-teste" (FAN, } \\
\text { XIAO, SU, 2015, p. 1223). } \\
\text { Aumento em 10\% no resultado do grupo experimental pós-teste em relação ao } \\
\text { pós-teste do grupo controle. }\end{array}$ \\
\hline 4 & BARATA et. al. & $\begin{array}{l}\text { "As notas finais foram significativamente diferentes" (BARATA et. al. } 2013 \text { p. } \\
\text { 14). } \\
\text { a nota mínima mais alta e a maioria dos alunos alcançando a nota mais alta de } \\
\text { todos os tempos, o que sugere uma melhoria nos resultados da aprendizagem. } \\
\text { Menor disparidade na distribuição de notas, o que parece ter beneficiado todos } \\
\text { os alunos, incluindo aqueles que obtiveram nota baixa. } \\
\text { Os alunos parecem ter uma melhor pontuação com a versão gamificada do } \\
\text { curso e as diferenças de notas entre eles parecem diminuir. }\end{array}$ \\
\hline
\end{tabular}

Fonte: Desenvolvido pelos autores.

Muito embora o foco principal do estudo fosse a análise de possíveis benefícios ao processo de aprendizagem, os artigos em questão também enfatizaram, em quase todos os resultados, outros pontos interessantes relacionados à promoção de um bem-estar expressado pelos próprios usuários, além de se apresentar uma ferramenta motivadora e que despertou a criatividade e o interesse nos alunos.

Observa-se também, como sugestão do estudo 3, uma aproximação das disciplinas do Design, dado o exemplo do Design Instrucional, como forma de mapear um processo de aprendizagem mais integralizado às necessidades e anseios dos alunos e professores.

Quadro 3: Resultados no tocante a outras descobertas relevantes para a revisão bibliográfica atual

\begin{tabular}{|l|l|}
\hline \multicolumn{1}{|c|}{$\begin{array}{c}\text { No do } \\
\text { Artigo }\end{array}$} & \multicolumn{1}{c|}{ Outros resultados encontrados } \\
\hline 1 & Não foram obtidos resultados extras. \\
\hline 2 & $\begin{array}{l}\text { "descobrimos que a gamificação pode se tornar um método importante para motivar o aluno e } \\
\text { despertar sua criatividade na solução de problemas complexos" (CABADA, 2018 p. 124). } \\
\text { "Os testes realizados mostraram que a detecção do estado emocional do aluno pode ter uma } \\
\text { influência favorável quando usada para realizar uma intervenção, caso o aluno precise, } \\
\text { redirecionando a atenção (quando o aluno está entediado) ou mostrando auxílios informativos } \\
\text { (Quando frustrados)" (idem ibidem). }\end{array}$ \\
\hline 3 & $\begin{array}{l}\text { É apontado no estudo como implicação a análise do Design para o processo cognitivo da } \\
\text { aprendizagem significativa por gamificação. } \\
\text { "no entanto, essa abordagem de aprendizado não é gerada automaticamente pelos alunos; em vez } \\
\text { disso, conta com o design instrucional e a orientação dos professores para que os alunos possam } \\
\text { entender ativa e completamente o esquema de aprendizado" (FAN, XIAO, SU. 2015 p. 1227). }\end{array}$ \\
\hline 4 & $\begin{array}{l}\text { "Os alunos consideraram o projeto da MCP mais motivador e interessante quando comparado a } \\
\text { outros cursos" (BARATA et. al. 2013, p. 16). }\end{array}$ \\
\hline
\end{tabular}

Fonte: Desenvolvido pelos autores.

Além dos resultados mostrados pelas pesquisas analisadas pela revisão bibliográica, durante a observação dos modelos teóricos encontrados, foi possível perceber que boa parte dos artigos selecionados se baseavam não apenas as Teorias Cognitivas Interacionistas e metodologias ativas de aprendizado - como a aprendizagem significativa de Ausubel (1963) por exemplo, como também a gamificação pelo ponto de vista do Design de Interfaces, com Deterding $(2011,2011,2012)$, Prensky (2001, 2003) e Ziecherman e Cunningham (2003). 
Vale ainda ressaltar que, durante a fundamentação teórica dos estudos observados, também foram apontados vários outros exemplos na literatura científica de uma contribuição da aplicação de gamificação em estudos em relação a outros aspectos cognitivos, emocionais e de engajamento, como o aumento do êxito na aprendizagem - presencial e à distância - (GARRISON, 2004), motivação (MCCLEAN, 2001), zelo durante tarefas (MORENO 2012), melhoria do processo de memorização (DRISKELL, WILLIS, COOPER, 1992), ensino, entretenimento, promoção de auto-dignidade e prática de habilidades (DEMPSEY et. al., 1996), desenvolvimento do estado de concentração intensa, denominado flow (CSIKSZENTMIHALYI, 1990) e melhoria no estado afetivo e emocional (LANDOWSKA, 2014).

\section{Considerações Finais}

A RBI desenvolvida neste estudo propôs conhecer um pouco mais sobre as ferramentas de gamificação e sua contribuição na aprendizagem e desempenho cognitivo de jovens.

Os resultados da revisão encontram-se positivos à questão de pesquisa estabelecida inicialmente. Em quase todos os resultados pode-se perceber um aumento significativo no número de acertos por partes dos participantes do estudo. Alguns casos ainda indicam melhorias voltadas ao engajamento, demonstração de interesse e melhoria do estado emocional desses mesmos participantes. Também se faz presente nos estudos uma nota reforçando a importância das investigações integrando disciplinas do Design voltadas ao esquema de aprendizado gamificado.

No tocante às limitações do estudo, muito embora os resultados coletados possuam dados relacionados a usuários matriculados em cursos de ensino médio e superior, em uma quantidade significativa dos estudos a idade desses estudantes não é revelada. Além disso, o estudo 4 resulta de uma análise do quadro geral de alunos ao longo de 5 anos, com turmas diferentes em cada ano, impossibilitando uma análise de progressão dos alunos enquanto turma, no entanto, proporcionando essa mesma investigação a nível institucional.

De maneira geral, fica evidente a oportunidade de melhorias no ensino de jovens através da implementação de plataformas online de aprendizagem gamificadas, podendo estas trazer benefícios não apenas em aspectos de resultados como também se tratando de bem estar e outros fatores cognitivos e psicológicos.

Também fica aberta como sugestão derivada deste artigo alguma possível a investigação de possíveis contribuições da aplicação desse modo de ensino em diferentes espectros e plataformas, incluindo o Design de interfaces e demais estudos cognitivos nas áreas da Ergonomia e do Design.

\section{Gamification and youth learning: RBI on Cognitive Aspects and Interface Design}

Abstract: This article discusses the importance of cognitive ergonomics in the creation of gamification tools and aims to discuss the effectiveness of its application in high school and higher education for young people, as well as its cognitive impact. The method used was the Integrative Bibliographic Review (RBI), carried out between 2010 and 2020, through the selection of four electronic bases, distributed among the groups of research sequences: Gamification + Impact Cognitive + Young. The four results obtained in the research - Silva, Sales and Castro (2019), Cabada (2018), Fan, Xiao and Su (2015) and Barata et. al. (2013) - makes it possible to understand or use gamification in learning platforms, their stimuli to increase the number of numbers and the other cognitive benefits brought by the insertion of these tools in the teaching context.

Keywords: Gamification; Interface Design; Cognitive ergonomics; Cognitive impact; Integrative bibliographic review 


\section{Referências bibliográficas}

4 sugestões de aulas interativas para utilizar em sala. Simulare. 2019. Disponível em:<https://simulare.com.br/blog/4-sugestoes-de-aulas-interativas-para-utilizar-em-sala/>. Acesso em 03 Jul. 2020.

ARREGUY, Juliana. Como criar um questionário no Kahoot. UmCOMO. Disponível em:< https://educacao.umcomo.com.br/artigo/como-criar-um-questionario-no-kahoot-27372.html>. Acesso em 14 set 2020.

AUSUBEL, David Paul. Psychology of Meaningful Verbal Learning: an introduction to school learning. New York: Grune \& Stratton, 1963.

BARATA, Gabriel.; GAMA, Sandra.; JORGE, Joaquim Armando Pires.; GONÇALVES, Daniel. Improving Participation and Learning with Gamification, in: Gamification 2013 - Proceedings, ACM, 2-4 out. 2013, p. 10-17.

BOTELHO, Louise Lira Roedel Botelho; CUNHA, Cristiano Castro de Almeida; MACEDO, Marcelo. O método de revisão integrativa nos estudos organizacionais, in: Gestão e sociedade, 5(11):121-136, 2011. https://doi.org/10.21171/ges.v5i11.1220.

CABADA, Ramón Zatarin. Reconocimiento afectivo y gamificación aplicados al aprendizaje de Lógica algorítmica y programación, in: Anais da Revista Electrónica de Investigación Educativa, [s.l.], v. 20, n. 3, p. 115-125, 7 set. 2018. http://dx.doi.org/10.24320/redie.2018.20.3.1636.

CSIKSZENTMIHALYI, Mihaly. Flow: The Psychology of Optimal Experience. HarperCollins, New York, 1990.

CSIKSZENTMIHALYI, Mihaly. Flow: The Psychology of Optimal Experience. Harper Perennial, New York, 1991.

DEMPSEY, John.; LUCASSEN, Barbara.; HAYNES, Linda.; CASEY, Maryann. Instructional applications of computer games. (ERIC Document Reproduction Service No. ED394500)., 1996.

DETERDING, Sebastian. Gamification: Designing for motivation, in: Interactions. [s.I.], v. 19, p. 1417, 2012. 10.1145/2212877.2212883.

DETERDING, Sebastian.; DIXON Dan.; KHALED Rilla.; NACKE, Lennart. From Game Design Elements to Gamefulness: Defining Gamification in: Atas do International Academic Mindtrek

Conference: Envisioning Future Media Environments. Tampere: 2011 (ACM, New York, 2011). p. 9.

DETERDING, Sebastian.; SICART, Miguel.; NACKE, Lennart.; O'HARA, Kenton.; DIXON, Dan.

Gamification: using game design elements in non-game contexts, in: Atas do Annual Conference

Extended Abstracts on Human Factors in Computing Systems. Vancouver, Canadá: 2011. doi:10.1145/1979742.1979575.

DRISKELL, James E.; WILLIS, Ruth. P.; COOPER, Carolyn. Effect of overlearning on retention in: Anais do Journal of Applied Psychology, 77, 615-622, 1992.

FAN, Kuo-Kuang.; XIAO, Peng-wei.; SU, Chungho. The Effects of Learning Styles and Meaningful Learning on the Learning Achievement of Gamification Health Education Curriculum, in: Anais do Eurasia Journal Of Mathematics, Science \& Technology Education, [s.l], v. 5, n. 11, p. 12111229, 24 jun. 2015.

GARRISON D. Randy; KANUKA Heather. Blended learning: Uncovering its transformative potential in higher education, in: Anais do The internet and higher education, 7(2):95- 105, 2004.

HUZINGA, Johan. Homo Ludens: O jogo como elemento da cultura. São Paulo: Perspectiva, 2018. LANDOWSKA, Agnieszka. (October, 2014). Affective learning manifesto -10 Years Later, in: Atas do 13th European Conference on e-Learning ECEL R. Ørngreen y K. T. Levinsen (Eds.), 2014. 
MCCLEAN, Phillip; SAINI-EIDU, Bernhardt; SCHWERT, Ddonald; SLATOR, Brian; WHITE, Alan. Virtual worlds in large enrollment science classes significantly improve authentic learning, in: Atas do 12th International Conference on College Teaching and Learning, [s.I.], 2001.

METHLEY, Abi; CAMPBELL, Stephen; CHEW-GRAHAM, Carolyn; MCNALLY, Rosalynd; CHERAGHI-SOHI, Sudeh. PICO, PICOS and SPIDER: a comparison study of specifitity and sensituvity in three search tools for qualitative systematic reviews, in: Atas do BMC Health Services Research v.14, [s.l.] 2014.

MORENO, Julián. Digital competition game to improve programming skills, in: Atas do Educational Technology \& Society, 15(3):288-297 [s.l.], 2012.

NORMAN, Donald. A. Design Emocional: Por que adoramos (ou detestamos) os objetos do dia a dia. Rio de Janeiro: Rocco, 2008.

PIAGET, Jean. Sobre a Pedagogia: textos inéditos. Org. Silvia Parrat e Anastásia Tryphon, São Paulo: Casa do Psicólogo, 1998.

PRENSKY, Marc. Digital Game-Based Learning. NY: McGraw-Hill, 2001.

PRENSKY, Marc. Digital game-based learning, in: Atas do Computer in Entertainment (CIE), 1(1),21 -21. [s.I.] 2003.

PRISMA. Disponível em:<http://www.prisma-statement.org/Default.aspx>. Acesso em 20 Jun 2020.

SILVA, João Batista da; SALES, Gilvandenys Leite; CASTRO, Juscileide Braga de. Gamificação como estratégia de aprendizagem ativa no ensino de Física, in: Anais da Revista Brasileira de Ensino de Física, v. 41, n. 4, 21. FapUNIFESP (SciELO). [s.I.], 2019. http://dx.doi.org/10.1590/1806-9126rbef-2018-0309.

SILVA, Rafael Silvério da. Perfil do aluno EAD no Brasil: as características de um estudante bemsucedido. EADBox, 2018. Disponível em:<https://eadbox.com/perfil-aluno-ead-

brasil/\#Dicas_para_se_dar_bem_no_ensino_EAD>. Acesso em 23 Jun 2020.

Uso de internet, televisão e celular no Brasil. IBGE Educa. Disponível em: <https://educa.ibge.gov.br/jovens/materias-especiais/20787-uso-de-internet-televisao-e-celularno-brasil.html>. Acesso em 10 Jul de 2020.

VYGOTSKY, Lev. Semionovitch. A formação Social da Mente: Desenvolvimento da Percepção e da Atenção. 6. ed. [S.I.]: Martins Fontes, 2003.

ZICHERMANN, Gabe.; CUNNINGHAM, Cristopher. Gamification by Design: Implementing Game Mechanics in Web and Mobile Apps. O'Reilly Media, Inc., 2011. 